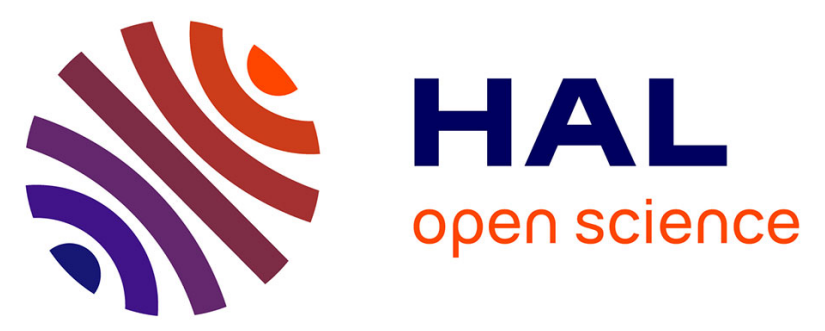

\title{
Inverse problem approach in Particle Digital Holography : out-of-field particle detection made possible
}

Ferréol Soulez, Loïc Denis, Éric Thiébaut, Corinne Fournier, Charles Goepfert

\section{- To cite this version:}

Ferréol Soulez, Loïc Denis, Éric Thiébaut, Corinne Fournier, Charles Goepfert. Inverse problem approach in Particle Digital Holography: out-of-field particle detection made possible. Journal of the Optical Society of America. A Optics, Image Science, and Vision, 2007, 24 (12), pp.3708-3716. 10.1364/JOSAA.24.003708 • ujm-00192619

\section{HAL Id: ujm-00192619}

\section{https://hal-ujm.archives-ouvertes.fr/ujm-00192619}

Submitted on 6 Jun 2008

HAL is a multi-disciplinary open access archive for the deposit and dissemination of scientific research documents, whether they are published or not. The documents may come from teaching and research institutions in France or abroad, or from public or private research centers.
L'archive ouverte pluridisciplinaire HAL, est destinée au dépôt et à la diffusion de documents scientifiques de niveau recherche, publiés ou non, émanant des établissements d'enseignement et de recherche français ou étrangers, des laboratoires publics ou privés. 


\title{
Inverse problems approach in Particle Digital Holography: out-of-field particle detection made possible
}

\author{
Ferréol Soulez, ${ }^{1,2, *}$ Loïc Denis, ${ }^{2,3}$ Éric Thiébaut, ${ }^{1}$ Corinne Fournier, ${ }^{2}$ and Charles Goepfert ${ }^{4}$ \\ 1 Université de Lyon, Lyon, F-69000, France; Université Lyon 1, \\ Villeurbanne, F-69622, France; Centre de Recherche Astronomique de Lyon, \\ Observatoire de Lyon, 9 avenue Charles André, \\ Saint-Genis Laval cedex, F-69561, France; CNRS, \\ UMR 5574; Ecole Normale Supérieure de Lyon, Lyon, France \\ 2 Laboratoire Hubert Curien (ex-LTSI) ; CNRS, \\ UMR5516 ; Université Jean Monnet; 18 rue Pr Benoît Lauras, F-42000 Saint-Etienne, France \\ 3 Signal and Image Processing Department, GET-Télécom Paris, \\ CNRS UMR 5141, 46 rue Barrault, 75013 Paris, France \\ 4 Laboratoire de Mécanique des Fluides et d'Acoustique, \\ CNRS UMR 5509 ; Ecole Centrale de Lyon; Université Lyon 1; INSA Lyon, \\ 36 avenue Guy-de-Collongue F-69134 ECULLY cedex, France and \\ *Corresponding author: ferreol.soulez@obs.univ-lyon1.fr
}

\begin{abstract}
This paper proposes a micro-particles detection scheme in digital holography. In our inverse problems approach, we estimate the optimal particles set which best models the observed hologram image. Such a method can deal with data which have missing pixels. By considering the camera as a truncated version of a wider sensor, it becomes possible to detect particles even out of the camera field of view. We tested the performances of our algorithm against simulated and experimental data for diluted particle conditions. With real data, our algorithm can detect particles far from the detector edges in a working area as large as 16 times the camera field of view. A study based on simulated data shows that, compared to classical methods, our algorithm greatly improves the precision of the estimated particle positions and radii. This precision does not depend on the particle's size nor location (i.e. inside or ouside the detector field of view).
\end{abstract}

(C) 2007 Optical Society of America

OCIS codes: $090.1760,100.3190,100.5010,100.6640,100.2000$

\section{Introduction}

Experimental flow has long been studied by imaging particles that are carried by the flow, either naturally present or artificially seeded. The associated techniques are known as Particle Tracking Velocimetry (PTV) when each particle is tracked from one image to another, and Particle Image Velocimetry (PIV) where local flow velocity is computed on a correlation criterion basis, suitable only for high particle concentrations. Velocity and size measurements are still often limited to the plane of the light sheet by the lack of accurate truly 3-D imaging techniques.

The 3-D recording capability of holography makes it suitable for 3-D particle imaging and a lot of work has been done in the field of particle holography [1-6]. Digital holography has greatly improved the processing of holograms by suppressing the wet-chemical processing step and opening new frontiers for automated volume analysis. Hologram analysis still remains largely influenced by traditional optical processing and most authors describe analysis of 3-D reconstructed volume obtained by diffraction simulation (Fresnel transform [1], fractional transforms[7] or wavelets transform[8, 9]). We refer to those approaches as classical approaches as they are widely used and accepted.

Classical digital holography is performed in two steps: first, a hologram is recorded with a holographic setup directly on a digital camera; then, this hologram is processed by reconstructing a $3-\mathrm{D}$ volume and analyzing the volume. In the case of particle holograms, the most efficient setup is the Gabor setup (Fig. 7) which requires few optical components: a coherent source, either a continuous laser or a pulsed laser synchronized with the camera, and a beam expander. The particles create a set of diffraction patterns named a Gabor hologram. The use of such a setup limits the particle size and the particle concentration to small particles whose projections on the sensor cover less than $1 / 100$ to $1 / 10$ of its surface [10]. The hologram is then processed in the second step by first reconstructing a volumetric image using diffraction simulation transforms (e.g. volume restitution by Fresnel transform of the zero padded hologram image); and then the reconstructed volume is segmented and the particle 3-D location and size can be deduced from the centroid and mean diameter of each segmented object. Such ap- 
proaches, however, suffer from various limitations: (i) the depth resolution is about 10 times worse than the transverse resolution; (ii) the field of view is limited and, in practice, must be restricted to the center of the hologram to reduce the border effects; (iii) spurious twin-images of the particles get reconstructed; (iv) multiple focusing[11] can occur around the actual depth location of each particle.

In a companion article[12], we recently suggested an inverse problems approach to overcome the issue of accurate location of particles. It however remains that digital holograms are today technologically limited to a few $\mathrm{cm}^{2}$, that are several times smaller than the size of holographic plates. Digital holograms therefore suffer from truncation which leads to strong artifacts in the reconstructed volume for particles found close to the borders of the hologram. Out-of-field particles (i.e. particles whose projection is outside the hologram) can not be recovered by classical techniques unless they are a few tenths of a pixels out of the hologram support.

Our preceding method detects particles in three steps: pre-localization using a classical algorithm, location refinement, and particle cleaning. The limitations of classical algorithms can be partially avoided by expressing the particle detection problem as a global optimization problem that is solved locally. However, the first step, based on classical algorithms, restricts the method to particles in the field of the hologram. This field is smaller in the case of digital holography than that of optical holography using a holographic plate and remains a drawback for flow measurements by digital holography. Since our previous algorithm is based on an inverse problems approach, it could be applied to out-of-field particles if only they could be detected in the first step of the method. In the present paper we propose to extend our inverse problems approach to the pre-localization stage of our method so as to achieve detection of out-of-field particles.

The paper is organized as follows. In Sect. 2, we briefly recall the general principle of our method[12] which detects particles in three steps: pre-localization using a classical algorithm, location refinement, and particle cleaning. In Sect. 3, we also recall the equations of the model of the hologram used in our simulations and in the inverse problems approach. In Sect. 4, we derive an alternative technique to perform the volume exploration. In Sect. 5, we present results obtained with our algorithm on both simulated and experimental data showing that out-of-field particle detection was effectively achieved with almost no loss in precision. The benefits of the new algorithm are summarized in Sect. 6 .

\section{General Algorithm Scheme}

The particle detection method presented here, takes place in a general particle localization scheme described in the companion article[12]. The different stages of this algorithm are depicted in Fig. 1. First, a detection algorithm gets an approximate location of a particle, then

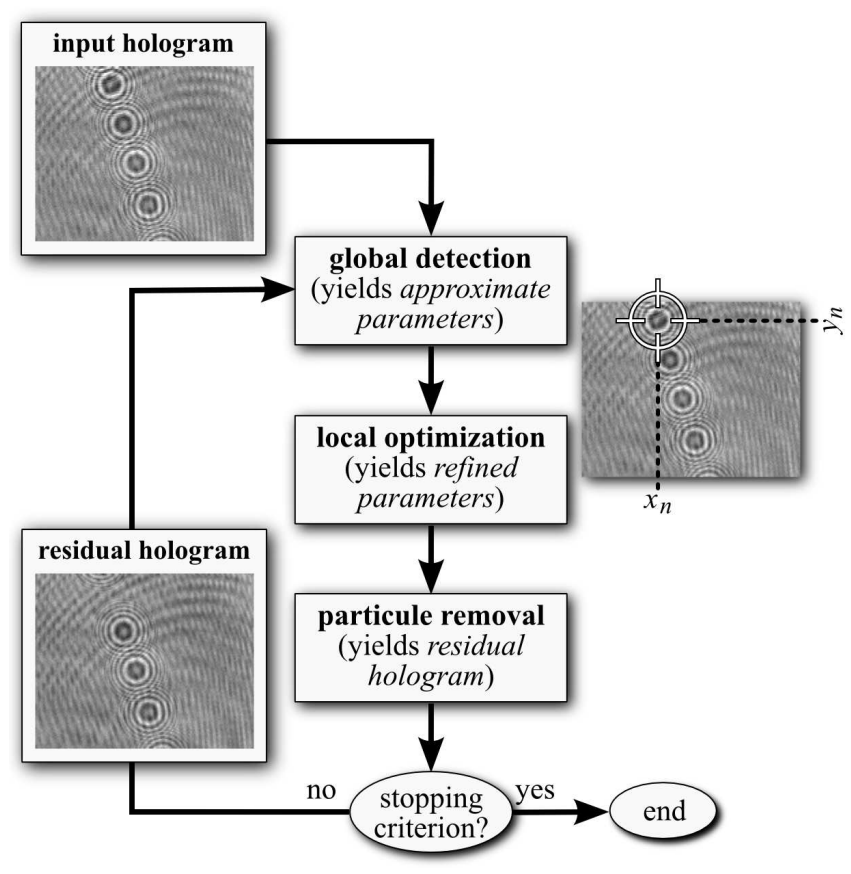

Fig. 1. Synopsis of the method.

this location is refined using an optimization procedure, and finally the diffraction pattern of the detected particle is subtracted from the hologram. The hologram is iteratively processed by the three steps and is gradually cleaned from all the previously detected particles. We stop the iterative method and consider that all particles have been detected when the next most likely particle found by the algorithm has aberrant parameters (negligible or negative contribution, too large or too small radius...).

We have shown on real data that the coarse localization step followed by the local optimization step effectively solve the global optimization problem of finding the best size and location of a particle in the (residual) hologram image, even for particles located near the hologram borders. Repeating the detection and localization steps after removal of previously detected particles, allows particles with fainter signatures to be found, in particular particles distant from the camera center.

Since our algorithm is derived following an inverse problems approach, it does not require any explicit direct inversion of the observed hologram: all estimations are made in the data space. As a result, our algorithm has a number of advantages over other existing methods. For instance, our method is insensitive to the twinimage problem inherent in in-line holography. Moreover, our algorithm is even able to deal with truncated field of view and with holograms with saturated or dead pixels. However, the first detection step (based on a classical approach) must be improved in order to detect out of field particles. 


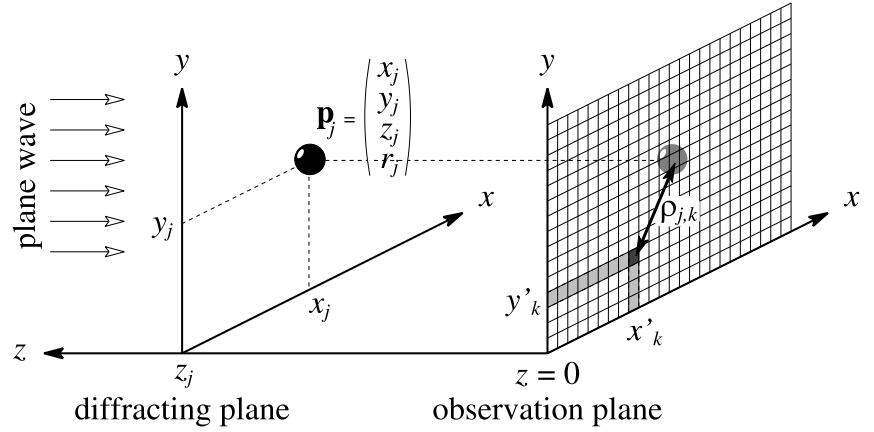

Fig. 2. Notations used in the hologram model. The parameters $\mathbf{p}_{j}=\left\{x_{j}, y_{j}, z_{j}, r_{j}\right\}$ are the position and radius of the $j$-th particle, $\left(x_{k}^{\prime}, y_{k}^{\prime}\right)$ is the location of the $k$-th pixel and $\rho_{j, k}$ is the distance between the projection of the $j$-th particle on the detector and the $k$-th pixel.

\section{Model of the hologram formation}

In this section, we recall the model of the observed holograms derived in our previous paper[12]. We consider an in-line holography setup (see Fig. 7) where studied particles are illuminated with a collimated laser beam, and the digital camera records both the object wave (scattered light) and the reference wave (laser). We work with small spherical particles and under Fresnel's diffraction approximation[13], that is for $z_{j}^{3} \gg 4 \pi r_{j}^{4} / \lambda$, which is verified for the conditions considered in our simulations and experimental results: laser wavelength $\lambda=532 \mathrm{~nm}$, particle distances to the detector $z_{j} \approx 250 \mathrm{~mm}$ and particle radii $r_{j} \lesssim 55 \mu \mathrm{m}$

In our previous paper[12], we have shown that, under such conditions and for $n$ particles of radii $r_{j}$ and $3-\mathrm{D}$ positions $\left(x_{j}, y_{j}, z_{j}\right)$, the intensity measured by the detector at position $(x, y)$ is given by:

$$
\begin{array}{r}
I(x, y)=I_{\mathrm{src}}+I_{\mathrm{bg}}-2 I_{\mathrm{src}} \sum_{j=1}^{n} \eta_{j} \operatorname{Re}\left(\underline{f_{j}}(x, y)\right) \\
+I_{\mathrm{src}} \sum_{i=1}^{n} \sum_{j=1}^{n} \eta_{i} \underline{f_{i}}(x, y) \eta_{j} \underline{f_{j}^{*}}(x, y)
\end{array}
$$

where $I_{\mathrm{src}}$ is the image level due to the laser source, $I_{\mathrm{bg}}$ is the background level, the real factors $\eta_{j} \in[0,1]$ account for possible variation of incident energy seen by a particle due to non-uniform laser illumination and $f_{j}$ is the amplitude diffraction term by $j$-th particle $[12,14]$ :

$$
\begin{gathered}
\underline{f_{j}}(x, y)=\frac{r_{j}}{2 \mathrm{i} \rho_{j}(x, y)} \mathrm{J}_{1}\left(\frac{2 \pi r_{j} \rho_{j}(x, y)}{\lambda z_{j}}\right) \\
\quad \times \exp \left(\mathrm{i} \frac{\pi \rho_{j}^{2}(x, y)}{\lambda z_{j}}\right)
\end{gathered}
$$

where $\mathrm{i}=\sqrt{-1}, \mathrm{~J}_{1}$ is the first order Bessel function and where $\rho_{j}(x, y)=\sqrt{\left(x-x_{j}\right)^{2}+\left(y-y_{j}\right)^{2}}$ is the distance between the point $(x, y)$ in the detector plane (at $z=0$ ) and the projection $\left(x_{j}, y_{j}\right)$ of the location of the $j$-th particle on the detector.

Under the considered conditions (low particle density, $z_{j} \approx 250 \mathrm{~mm}$ and $r_{j} \lesssim 55 \mu \mathrm{m}$ ), the second order interference terms in Eq. (1) can be neglected[12] and the model simplifies to:

$$
I(x, y) \simeq I_{0}-\sum_{j=1}^{n} \alpha_{j} g_{j}\left(x-x_{j}, y-y_{j}\right)
$$

with $I_{0}=I_{\mathrm{src}}+I_{\mathrm{bg}}, \alpha_{j}=2 I_{\mathrm{src}} \eta_{j}$ and:

$$
\begin{aligned}
g_{j}(\Delta x, \Delta y)= & \frac{r_{j}}{2 \rho(\Delta x, \Delta y)} \mathrm{J}_{1}\left(\frac{2 \pi r_{j} \rho(\Delta x, \Delta y)}{\lambda z_{j}}\right) \\
& \times \sin \left(\frac{\pi \rho^{2}(\Delta x, \Delta y)}{\lambda z_{j}}\right)
\end{aligned}
$$

where $\rho(\Delta x, \Delta y)=\sqrt{\Delta x^{2}+\Delta y^{2}}$ is the projected distance.

The model in Eq. (3) will be the basis of our particle detection approach in Sec. 4. The resulting hologram expression is a sum of terms depending on the location and size of each diffracting particle. The notations and coordinate system used in our model are summarized in Fig. 2.

\section{Particle detection}

Assuming this model of hologram formation, we suggest in this section a new particle detection scheme achieving detection of out of field particles. Determining the optimal set of particles that best models the intensity distribution of the hologram is equivalent to finding the $n$ particle parameters $\left\{x_{j}, y_{j}, z_{j}, r_{j} ; j=1, \ldots, n\right\}$ that minimize the weighted least-squares penalty:

$$
\mathcal{P}_{n}=\sum_{x_{k}^{\prime}} \sum_{y_{k}^{\prime}} w\left(x_{k}^{\prime}, y_{k}^{\prime}\right)\left[m_{n}\left(x_{k}^{\prime}, y_{k}^{\prime}\right)-d\left(x_{k}^{\prime}, y_{k}^{\prime}\right)\right]^{2}
$$

where $\left(x_{k}^{\prime}, y_{k}^{\prime}\right)$ are the coordinates of the $k$-th pixel, $m_{n}$ is the model, $d\left(x_{k}^{\prime}, y_{k}^{\prime}\right)$ is the $k$-th pixel value in the observed hologram and $w\left(x_{k}^{\prime}, y_{k}^{\prime}\right)$ is its statistical weight:

$w\left(x_{k}^{\prime}, y_{k}^{\prime}\right)= \begin{cases}\frac{1}{\operatorname{Var}\left(d\left(x_{k}^{\prime}, y_{k}^{\prime}\right)\right)} & \text { if } k \text {-th pixel is measured } \\ 0 & \text { otherwise. }\end{cases}$

where $\operatorname{Var}\left(d\left(x_{k}^{\prime}, y_{k}^{\prime}\right)\right)$ is the noise variance at pixel $\left(x_{k}, y_{k}\right)$. The noise considered here is meant to account for the detector noise and for the photon noise but $\operatorname{Var}\left(d\left(x_{k}^{\prime}, y_{k}^{\prime}\right)\right)$ can also be set to account for artifacts (such as fringes due to the detector glass window) and for approximation errors (such as digitization and such as speckle noise due to undetected objects at this stage or due to the neglected interferences between particles).

In the case where the noise is assumed constant all over the known pixels, $\frac{1}{\operatorname{Var}\left(d\left(x_{k}^{\prime}, y_{k}^{\prime}\right)\right)}$ can be factorized out 
of Eq. (5). In this case $w\left(x_{k}, y_{k}\right)$ can be viewed as a binary mask on the data:

$$
w\left(x_{k}^{\prime}, y_{k}^{\prime}\right)= \begin{cases}1 & \text { if } k \text {-th pixel is measured } \\ 0 & \text { otherwise }\end{cases}
$$

The model $m_{n}\left(x_{k}^{\prime}, y_{k}^{\prime}\right)$ for $n$ particles is given by Eq. (3):

$$
m_{n}\left(x_{k}^{\prime}, y_{k}^{\prime}\right)=I_{0}-\sum_{j=1}^{n} \alpha_{j} g_{j}\left(x_{j}-x_{k}^{\prime}, x_{j}-y_{k}^{\prime}\right)
$$

As our algorithm proceeds iteratively for each particle (as described in Fig. 1), we consider the previously detected particles parameters as fixed. In our iterative approach, locating the $n$-th particle consists of determining the parameters of the $n$-th particle and the constant level that minimizes $\mathcal{P}_{n}$. This is achieved by fitting the parameters $\left\{\alpha_{n}, I_{0}, x_{n}, y_{n}, z_{n}, r_{n}\right\}$ on the centered residual image $r_{n-1}$ :

$r_{n-1}\left(x_{k}^{\prime}, y_{k}^{\prime}\right)=d\left(x_{k}^{\prime}, y_{k}^{\prime}\right)+\sum_{j=1}^{n-1} \alpha_{j} g_{j}\left(x_{j}-x_{k}^{\prime}, y_{j}-y_{k}^{\prime}\right)-c_{n-1}$,

which is the hologram image after having subtracted the contribution of the previous $n-1$ particles and a constant $c$ defined as the mean gray level of the $(n-1)$-th residual image: and where:

$$
\begin{aligned}
\sigma_{w} & =\sum_{x_{k}^{\prime}} \sum_{y_{k}^{\prime}} w\left(x_{k}^{\prime}, y_{k}^{\prime}\right) \\
\sigma_{w r^{2}} & =\sum_{x_{k}^{\prime}} \sum_{y_{k}^{\prime}} w\left(x_{k}^{\prime}, y_{k}^{\prime}\right) r_{n-1}^{2}\left(x_{k}^{\prime}, y_{k}^{\prime}\right)
\end{aligned}
$$

$$
\begin{aligned}
\sigma_{w r g} & =\sum_{x_{k}^{\prime}} \sum_{y_{k}^{\prime}} w\left(x_{k}^{\prime}, y_{k}^{\prime}\right) r_{n-1}\left(x_{k}^{\prime}, y_{k}^{\prime}\right) g_{n}\left(x_{n}-x_{k}^{\prime}, y_{n}-(y, 6)\right. \\
\sigma_{w g} & =\sum_{x_{k}^{\prime}} \sum_{y_{k}^{\prime}} w\left(x_{k}^{\prime}, y_{k}^{\prime}\right) g_{n}\left(x_{n}-x_{k}^{\prime}, y_{n}-y_{k}^{\prime}\right) \\
\sigma_{w g^{2}} & =\sum_{x_{k}^{\prime}} \sum_{y_{k}^{\prime}} w\left(x_{k}^{\prime}, y_{k}^{\prime}\right) g_{n}^{2}\left(x_{n}-x_{k}^{\prime}, y_{n}-y_{k}^{\prime}\right)
\end{aligned}
$$

\section{A. Optimal Parameters Determination}

Given $x_{n}, y_{n}, z_{n}$ and $r_{n}$, the optimal offset $I_{n}^{+}$and factor $\alpha_{n}^{+}$for such a model are obtained by solving the linear system:

$$
\left\{\begin{array}{l}
\left.\frac{\partial \mathcal{P}_{n}}{\partial I_{n}}\right|_{I_{n}=I_{n}^{+}, \alpha_{n}=\alpha_{n}^{+}}=0 \\
\left.\frac{\partial \mathcal{P}_{n}}{\partial \alpha_{n}}\right|_{I_{n}=I_{n}^{+}, \alpha_{n}=\alpha_{n}^{+}}=0
\end{array}\right.
$$

which yields:

$$
\begin{aligned}
I_{n}^{+} & =\frac{-\sigma_{w g} \sigma_{w r g}}{\sigma_{w} \sigma_{w g^{2}}-\sigma_{w g}^{2}}=\alpha_{n}^{+} \frac{\sigma_{w g}}{\sigma_{w}}, \\
\alpha_{n}^{+} & =\frac{-\sigma_{w} \sigma_{w r g}}{\sigma_{w} \sigma_{w g^{2}}-\sigma_{w g}^{2}},
\end{aligned}
$$

$c_{n-1}=\sum_{x_{k}^{\prime}} \sum_{y_{k}^{\prime}} w\left(x_{k}^{\prime}, y_{k}^{\prime}\right)\left(d\left(x_{k}^{\prime}, y_{k}^{\prime}\right)+\sum_{j=1}^{n-1} \alpha_{j} g_{j}\left(x_{j}-x_{k}^{\prime}, y_{j}-y_{k}^{\prime}\right)\right)$ Replaci

In other words, this constant is such that: in Eq. (13) yields the partially optimized criterion:

$$
\sigma_{w r} \stackrel{\text { def }}{=} \sum_{x_{k}^{\prime}} \sum_{y_{k}^{\prime}} w\left(x_{k}^{\prime}, y_{k}^{\prime}\right) r_{n-1}\left(x_{k}^{\prime}, y_{k}^{\prime}\right)=0
$$

which is used to simplify the equations in what follows.

For the detection/location of the $n$-th particle, our misfit criterion writes:

$$
\begin{array}{r}
\mathcal{P}_{n}=\sum_{x_{k}^{\prime}} \sum_{y_{k}^{\prime}} w\left(x_{k}^{\prime}, y_{k}^{\prime}\right)\left[I_{n}-\alpha_{n} g_{n}\left(x_{n}-x_{k}^{\prime}, x_{n}-y_{k}^{\prime}\right)\right. \\
\left.-r_{n-1}\left(x_{k}^{\prime}, y_{k}^{\prime}\right)\right]^{2}
\end{array}
$$

with $I_{n}=I_{0}-c_{n-1}$. For the first particle, we take $r_{0}\left(x_{k}^{\prime}, y_{k}^{\prime}\right)=d\left(x_{k}^{\prime}, y_{k}^{\prime}\right)$, and $c_{0}=$ $\sum_{x_{k}^{\prime}} \sum_{y_{k}^{\prime}} w\left(x_{k}^{\prime}, y_{k}^{\prime}\right) d\left(x_{k}^{\prime}, y_{k}^{\prime}\right)$. The expression in Eq. (12) can be expanded as:

$\mathcal{P}_{n}=\sigma_{w r^{2}}+2 \alpha_{n} \sigma_{w r g}-2 \alpha_{n} I_{n} \sigma_{w g}+I_{n}^{2} \sigma_{w}+\alpha_{n}^{2} \sigma_{w g^{2}}$

where it is accounted for the fact that $\sigma_{w r}=0$ (see 11)

$$
\left.\mathcal{P}_{n}^{+} \stackrel{\text { def }}{=} \mathcal{P}_{n}\right|_{I_{n}=I_{n}^{+}, \alpha_{n}=\alpha_{n}^{+}}=\sigma_{w r^{2}}-\frac{\sigma_{w}\left(\sigma_{w r g}\right)^{2}}{\sigma_{w} \sigma_{w g^{2}}-\sigma_{w g}^{2}} .
$$

It remains to optimize $\mathcal{P}_{n}^{+}$with respect to the other parmaters, namely $x_{n}, y_{n}, z_{n}$ and $r_{n}$. This is equivalent to maximizing:

$$
\mathcal{Q}_{n} \stackrel{\text { def }}{=} \frac{\sigma_{w}\left(\sigma_{w r g}\right)^{2}}{\sigma_{w} \sigma_{w g^{2}}-\sigma_{w g}^{2}}
$$

under the condition (by definition $\alpha_{j}=2 \gamma\left|\underline{A_{0}}\right|^{2} \eta_{j}>$ $0)$ :

$$
\alpha_{n}^{+}=\frac{-\sigma_{w} \sigma_{w r g}}{\sigma_{w} \sigma_{w g^{2}}-\sigma_{w g}^{2}}>0
$$

\section{B. Fast Computation}

It is possible to achieve fast determination at pixel resolution of the projected coordinates $\left(x_{n}^{\prime}, y_{n}^{\prime}\right)$ of the $n$-th particle given its other parameters $z_{n}$ and $r_{n}$. The terms $\sigma_{w r g}, \sigma_{w g^{2}}$ and $\sigma_{w g}$ (given respectively by equations 
Eq. (16), Eq. (17) and Eq. (18)) are involved in the expression of the criterion $\mathcal{Q}_{n}$ to maximize. When $z_{n}$ and $r_{n}$ are chosen, these terms are simply $2-\mathrm{D}$ discrete convolutions (the term $\sigma_{w}$ is a constant which can be computed once for all). Under circulant approximation (as CCD is a rectangular grid), it is possible to compute these terms by a few fast Fourier transforms (FFT's) for any projected coordinates $\left(x_{n}, y_{n}\right)$ matching the locations of the CCD pixels. In other words, given the depth $z_{n}$ and radius $r_{n}$ of the $n$-th particle, it is possible to quickly compute a 2-D map of the criterion $\mathcal{Q}_{n}$ with respect to the projected coordinates and with a resolution equal to the size of a pixel. The maximum of this map indicates the most likely projected positions of the $n$-th particle if it were located at distance $z_{n}$ with a radius $r_{n}$. In the following, we detail the computation of the 2-D map of $\mathcal{Q}_{n}$.

We denote $\stackrel{g}{g}$ the vector of values of $g_{n}$ for given depth $z_{n}$ and radius $r_{n}$ and sampled at projected coordinates equal to the CCD coordinates:

$$
\stackrel{\circ}{g}_{k} \stackrel{\text { def }}{=} g_{n}\left(x_{k}^{\prime}, y_{k}^{\prime}\right) \text {. }
$$

Similarly we denote by $\stackrel{\circ}{r}$ the $2-\mathrm{D}$ map of residuals:

$$
\dot{\circ}_{k} \stackrel{\text { def }}{=} \begin{cases}r_{n-1}\left(x_{k}^{\prime}, y_{k}^{\prime}\right) & d_{k} \text { is measured } \\ 0 & \text { otherwise }\end{cases}
$$

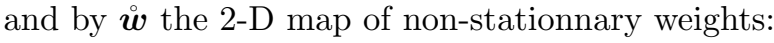

$\stackrel{\circ}{w}_{k} \stackrel{\text { def }}{=} \begin{cases}\operatorname{Var}\left(d_{k}\right)^{-1} & d_{k} \text { is measured, } \\ 0 & \text { otherwise (mainly outside the field of view) }\end{cases}$

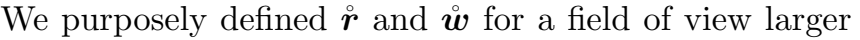
than the CCD support. The next section explains how support and aliasing issues are solved by using such an extended field of view. Note that the value of $\dot{\boldsymbol{r}}$ outside the field of view is irrelevant that's why the weights $\stackrel{\boldsymbol{w}}{ }$ must be set to zero outside the field of view (and for any bad pixels).

Under the circulant approximation, the 2-D map of $\sigma_{w g}$ can be approximated by:

$$
\stackrel{\circ}{\boldsymbol{a}} \stackrel{\text { def }}{=} \mathbf{F}^{-1} \cdot \operatorname{diag}(\mathbf{F} \cdot \stackrel{\leftrightarrow}{\boldsymbol{w}}) \cdot \mathbf{F} \cdot \stackrel{\circ}{\boldsymbol{g}}
$$

where $\mathbf{F}$ is the 2-D discrete Fourier transform (DFT) matrix operator and where $\operatorname{diag}(\boldsymbol{u})$ is the diagonal matrix with diagonal elements equal to the elements of vector $\boldsymbol{u}$; hence $\operatorname{diag}(\boldsymbol{u}) \cdot \boldsymbol{v}$ is simply the element-wise product of vectors $\boldsymbol{u}$ and $\boldsymbol{v}$ :

$$
[\operatorname{diag}(\boldsymbol{u}) \cdot \boldsymbol{v}]_{k}=u_{k} v_{k}
$$

Under circulant approximation, $\stackrel{a}{a}$ represents the correlation between $\stackrel{\circ}{\boldsymbol{w}}$ and $\stackrel{\circ}{\boldsymbol{g}}$. Likewise, the 2-D maps of $\sigma_{w r g}$ and $\sigma_{w g^{2}}$ can be respectively approximated by:

$$
\begin{aligned}
& \stackrel{\circ}{\boldsymbol{b}} \stackrel{\text { def }}{=} \mathbf{F}^{-1} \cdot \operatorname{diag}(\mathbf{F} \cdot \operatorname{diag}(\stackrel{\circ}{\boldsymbol{w}}) \cdot \stackrel{\circ}{\boldsymbol{r}}) \cdot \mathbf{F} \cdot \stackrel{\circ}{\boldsymbol{g}}, \\
& \stackrel{\circ}{\boldsymbol{c}} \stackrel{\text { def }}{=} \mathbf{F}^{-1} \cdot \operatorname{diag}(\mathbf{F} \cdot \stackrel{\circ}{\boldsymbol{w}}) \cdot \mathbf{F} \cdot \operatorname{diag}(\stackrel{\circ}{\boldsymbol{g}}) \cdot \stackrel{\circ}{\boldsymbol{g}} \text {. }
\end{aligned}
$$

Finally, the 2-D map of the criterion $\mathcal{Q}_{n}$ is approximated by:

$$
\mathcal{Q}_{k} \approx \stackrel{\circ}{\mathcal{Q}}_{k}=\frac{\sigma_{w} \stackrel{\circ}{b}_{k}}{\sigma_{w} \stackrel{\circ}{c}_{k}-\stackrel{\circ}{a}_{k}^{2}}
$$

which is the value of $\mathcal{Q}_{n}$ for a projected position $\left(x_{n}, y_{n}\right)=\left(x_{k}^{\prime}, y_{k}^{\prime}\right)$.

For every particle and for every sampled depth and particle radius, a circulant approximation of the 2-D map of $\mathcal{Q}_{n}$ must be computed. This requires 7 FFT's ( 4 direct FFT's plus 3 inverse FFT's). In fact, the DFT of $\boldsymbol{w}$ can be computed once for all, whereas the DFT of $\operatorname{diag}(\stackrel{\boldsymbol{w}}{)}) \cdot \stackrel{\circ}{\boldsymbol{r}}$ must be computed for every new particle, hence only 5 more FFT's are required for every particle and for every sampled depth and particle radius.

\section{Reconstruction Outside of the Field of View}

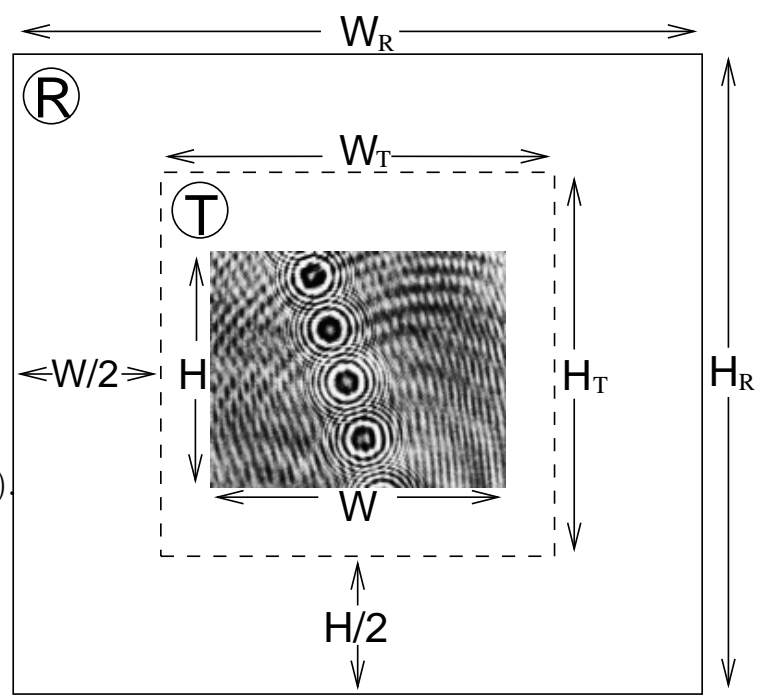

Fig. 3. Computing region $\mathrm{R}$ and working area $\mathrm{T}$ compared to the CCD size $(\mathrm{W} \times \mathrm{H})$

With such a formulation, it is possible to seek for particles outside of the field of view in a working area $\mathrm{T}$ which is larger than the CCD. However, for fast computation of $\mathcal{P}_{n}$, we use fast Fourier transforms which yield spatial aliasing because of the circulant approximation. To limit the aliasing effects, we perform all computations on a region $\mathrm{R}$ which is larger than the working area $\mathrm{T}$ where particles are to be detected. The width and height of the region $\mathrm{R}$ are chosen so that $W_{\mathrm{R}} \geq W_{\mathrm{T}}+W$ and $H_{\mathrm{R}} \geq H_{\mathrm{T}}+H$ where $H_{\mathrm{T}} \times W_{\mathrm{T}}$ and $W \times H$ are the dimensions of the working area $\mathrm{T}$ and of the detector (cf. Fig. 3). The residuals and statistical weights over the region $\mathrm{R}$ are set according to Eq. (25) and Eq. (26).

\section{Segmentation}

Laterally, $\mathcal{P}_{n}$ is sampled with the same size as pixel width inside and outside of the field of view. $\mathcal{P}_{n}$ is computed 
for several slices $\left(z_{n}, r_{n}\right)$ with a coarse sampling (typically every $\mathrm{mm}$ in depth and every $5 \mu \mathrm{m}$ for radius). This four dimensional space of solutions is exhaustively explored in order to extract parameters $\left\{x_{n}^{\prime+}, y_{n}^{\prime+}, z_{n}^{+}, r_{n}^{+}\right\}$that minimize $\mathcal{P}_{n}$. In practice, to reduce computation, only a three dimensional space of solutions $\left(x^{\prime}, y^{\prime}, z\right)$ is evaluated using an average radius. For improved efficiency, the mean radius can be estimated using the direct hologram analysis described in reference [15]. Eventually, at the optimal depth $z_{n}^{+}, \mathcal{P}_{n}$ can be computed for a few $r_{n}$ values depending on particle radius dispersion (see Sec. 5).

\section{Results}

\section{A. Simulated Data}

To assess the performance of our algorithm, we first processed simulated data. We compare the results obtained with the new algorithm and the results obtained with a classical approach. A study of the accuracy versus transversal location of the particle was conducted. This data set is composed of 32 holograms simulated with the same experimental conditions. Each hologram records the diffraction pattern of 20 disks with random radii between 40 and $50 \mu \mathrm{m}$ and randomly distributed in a box of $13.72 \times 13.72 \times 50.00 \mathrm{~mm}^{3}$. The wavelength of the laser is $0.532 \mu \mathrm{m}$. The camera has $1024 \times 1024$ pixels spaced by $6.7 \mu \mathrm{m}$ and is placed at about $250 \mathrm{~mm}$ from the studied volume in order to stay in the approximation range defined in section 3 . The field of view of this camera $\left(6.86 \times 6.86 \mathrm{~mm}^{2}\right)$ represents a quarter of the section of the studied volume and is centered on it.

Our inverse problems approach is based on a simplified model of the hologram image formation. To account for the effects of such simplifications on the detection and localization of the particles, we use a more accurate model to simulate the hologram images used in this test. More precisely, we use the image model given by Eq. (1) to simulate the holograms whereas the simplified model in Eq. (3) is assumed during the detection and localization steps of the algorithm. Hence the interference pattern between waves diffracted by different particles is accounted for in the simulated images whereas it is neglected by the algorithm. In order to properly simulate quantization by the detector, the dynamics of the simulated hologram images is converted into 8-bit integer values prior to their processing.

In the particle detection step, a reconstruction every millimeter in $z$ was sufficient for a successful coarse detection. These 50 detections were performed for an average radius of $45 \mu \mathrm{m}$. At the depth $z^{+}$that minimizes $\mathcal{P}_{n}, 5$ detections were performed for equally sampled radii between 40 and $50 \mu \mathrm{m}$. This leads to $(55 \times 5+2)$ FFT computations per particle.

The results of this inverse problems approach were compared with a classical particle detection algorithm (based on hologram reconstruction followed by 3-D segmentation). As such conventional methods can only deal with particles inside (or very close to) the camera field of view, we conducted the tests on simulated holograms under exactly the same conditions to have approximately the same number of detected particles. To achieve meaningful statistical results, we performed all the tests on 100 simulated holograms.

Our method detected almost every particle: on average, only 20 particles out of 640 were not detected (detection ratio of $97 \%$ ) and 2 were falsely detected (false positive ratio of $0.3 \%$ ). The conventional method detects 631 particles out of 2000 (detection ratio of $32 \%$ ). As expected, we noticed that such a method cannot properly deal with out of field particules (detection ratio of $100 \%$ in the field of view and $6 \%$ outside).

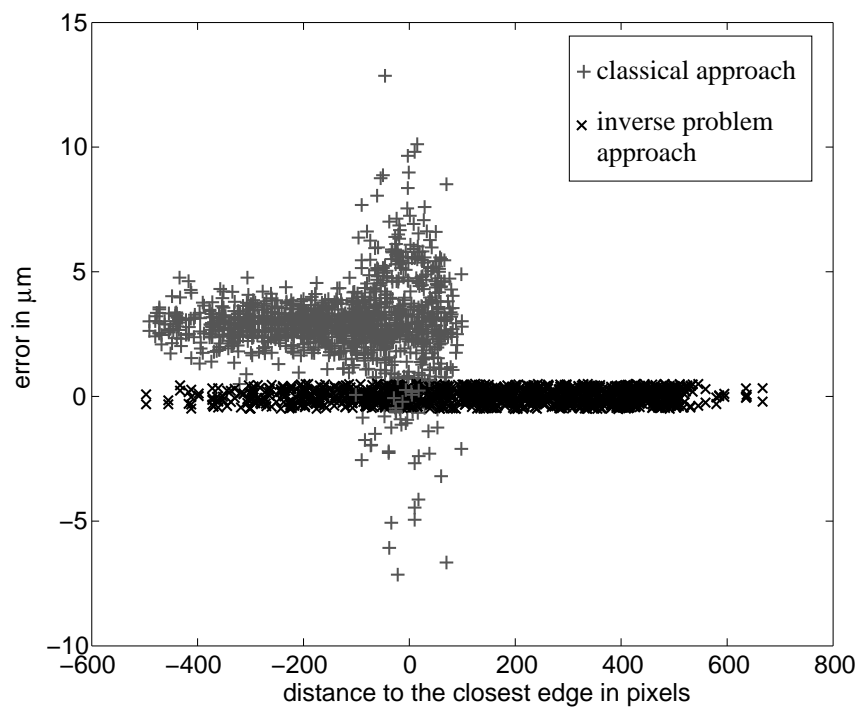

Fig. 4. Comparison of lateral error (on $\mathrm{x}$ and $\mathrm{y}$ ) for classical and "inverse problems" approach (pixel size: $6.7 \mu \mathrm{m}$ ).

Errors on particle parameters $(x, y, z, r)$ are plotted as a function of the distance of the particle to the closest edge of the CCD. This representation seems to underline well algorithm artifacts. Results are shown in Fig. 4, Fig. 5 and Fig. 6.

- Transversal errors of both methods are plotted in Fig. 4. Sub-pixel precision is reached for both methods but our method is unbiased and more precise. It presents an equal standard deviation on both $x$ and $y$ axis $(\Delta x \simeq \Delta y \simeq 0.3 \mu \mathrm{m}$ or $1 / 20$ pixel). This standard deviation does not significantly depend on the size nor the location of the particles.

- Fig. 5 shows the depth error for both methods. Our method improves greatly the classical one: the bias is negligible and the sub-pixel standard deviation ( $\Delta z \simeq 0.9 \mu \mathrm{m}$ or $1 / 7 \mathrm{pixel}$ ) is almost constant whatever the particle parameter.

- Only errors on radius for our approach are plotted in Fig. 6 as radius estimation by the classical 


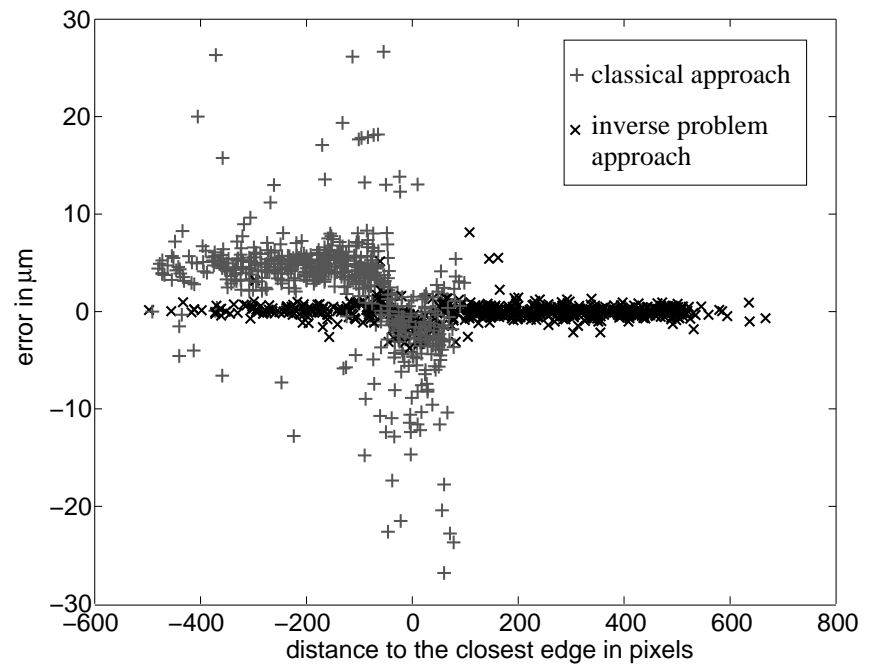

Fig. 5. Comparison of depth error for classical and "inverse problems" approach (pixel size: $6.7 \mu \mathrm{m}$ ).

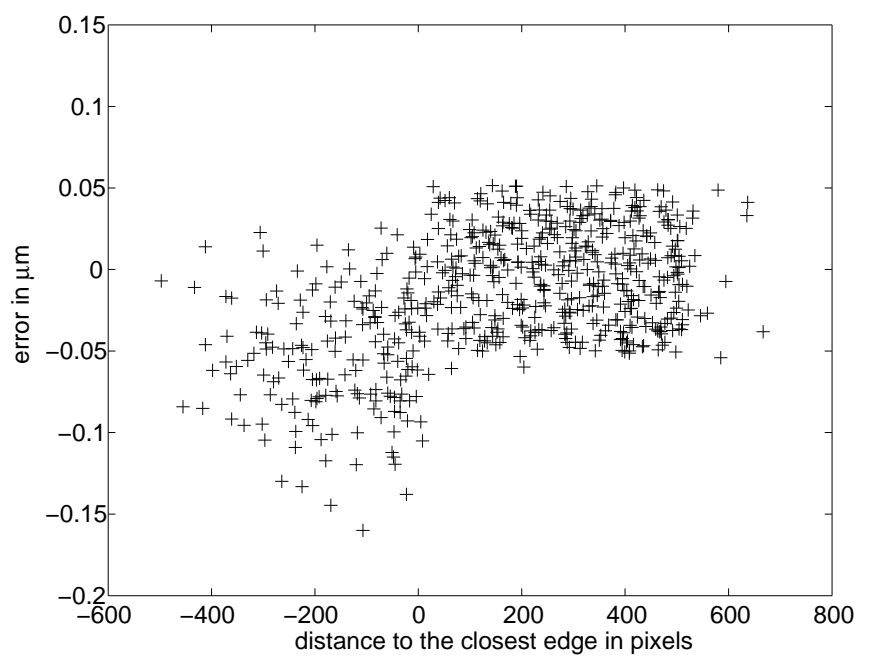

Fig. 6. Error on radius for the "inverse problems" approach (pixel size: $6.7 \mu \mathrm{m}$ ).

method presents errors too large to be plotted on the same figure (bias is $-8.8 \mu \mathrm{m}$ and $\Delta r \simeq 1.8 \mu \mathrm{m}$ for classical method). Radius estimation by our method is very precise with a standard deviation of $\Delta r \simeq 0.04 \mu \mathrm{m}$ for an average radius of $45 \mu \mathrm{m}$. Although radius estimation is unbiased for particles outside of the field of view, a small negative bias can be noticed for particles inside. This bias is due to the simplifications made in our model: the neglected interference term (second term in Eq. (1)) is larger when particles are inside of the field of view.

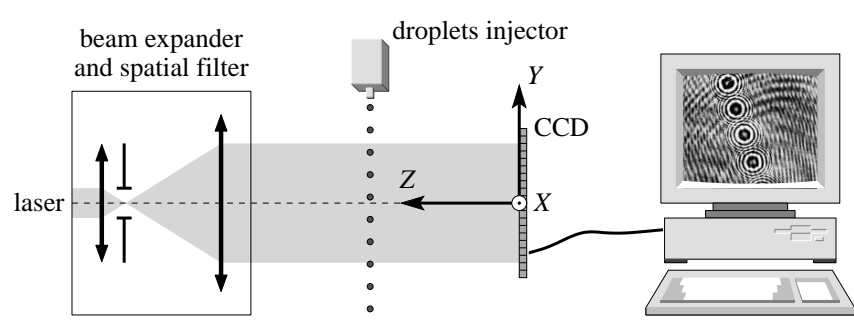

Fig. 7. In-line holography setup

\section{B. Experimental Data}

We carried out an experimental test of our algorithm using real data from an in-line holography setup. The experimental test data are the same as in the companion article[12]. The experimental layout is shown by Fig. 7 . The camera is a 12 -bit CCD with $1280 \times 1024$ pixels of size $6.7 \mu \mathrm{m} \times 6.7 \mu \mathrm{m}$. It is located at about $25 \mathrm{~cm}$ from the injector in order not to disturb the flow experiment. This leads to a small but realistic numerical aperture of $\Omega=0.014$. The laser wavelength is $0.532 \mu \mathrm{m}$. Studied particles are water droplets injected by a piezoelectric injector (conceived by Bremen University) which generates monodisperse droplets. Their sizes are adjustable between 50 to $100 \mu \mathrm{m}$.

The experimental dataset consists of a set of 100 pairs of holograms with four or five droplets on each image. An example of such holograms can be seen in the central box of Fig. 9. The 3-D locations and radii of droplets were extracted from this dataset by our algorithm. Each hologram was processed as described Sec. 2. At each iteration, $\mathcal{P}$ is computed to detect the next particle. Paste-up Fig. 8 shows an example of a slice $(x, y)$ with calculated $\mathcal{P}$ at each iteration. Fig. 9 illustrates the result of the processing of one hologram of the dataset. Let us point out that although four particles were in the camera field of view (in the box on the figure), up to 18 were detected.

The measured particles locations are shown in Fig. 10. The average trajectory clearly is close to the straight line expected from the experimental conditions. Detected out-of-field particle locations and their spacings describes well their ballistic trajectory. At each extremity of the reconstructed jet, the low signal to noise ratio increases the observed deviations from the ideal straight trajectory. For particles far from sensor edges, assumptions made in Sec. 3 become less relevant and model noise is added to measurement noise. The jet divergence which can be seen in Fig. 10 is mostly due to vibrations of the injector[12]. Hence, the effective precision of the measured locations is smaller than the variations due to these physical effects.

The droplet sizes estimated by our algorithm have a bell-shaped distribution (see Fig. 11) with a mean diameter of $94.1 \mu \mathrm{m}$ and a standard deviation of $0.3 \mu \mathrm{m}$ in agreement with the settings of the droplet injector. 


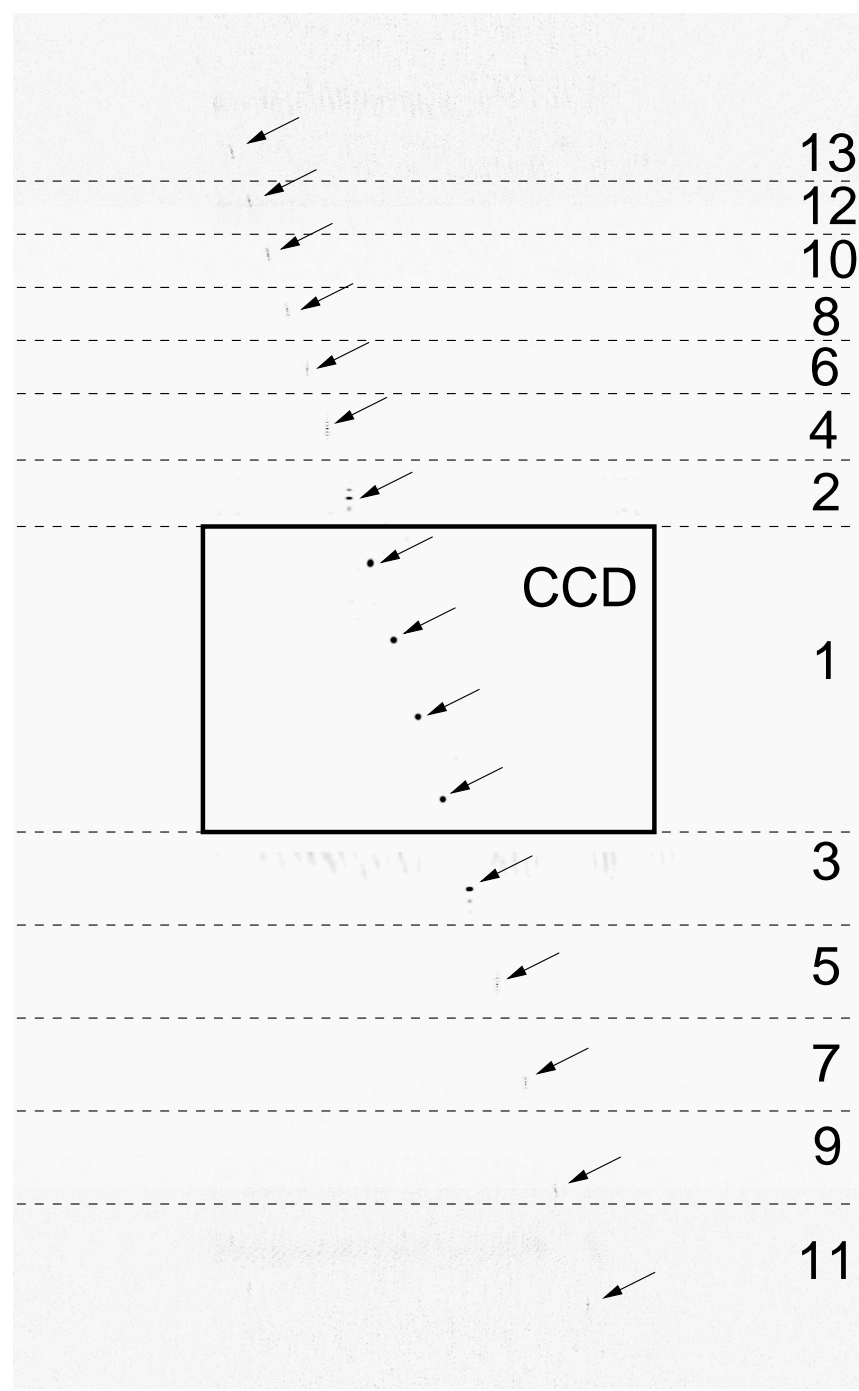

Fig. 8. Paste up representing a slice $(x, y)$ of $\mathcal{P}$ computed on an experimental hologram. It is composed of 13 images (numbered from 1 to 13). Each image is computed on the residuals after the detection of the particles (pointed by arrows) detected in the previous images.

\section{Conclusion}

In a companion article[12], we have introduced a new algorithm that accurately estimates the parameters of particles in digital holography. In this paper, we complete this scheme by describing a new algorithm for the detection of particles outside of the field of view of the sensor. The most important difference from other existing techniques is that our processing is based on an inverse problems approach and thus does not require any direct inversion. In this framework, we introduce a simplified model of the hologram images which directly incorporates the sizes and locations of the diffracting particles. We solve the problem by determining the set of particle parameters for which the difference between the

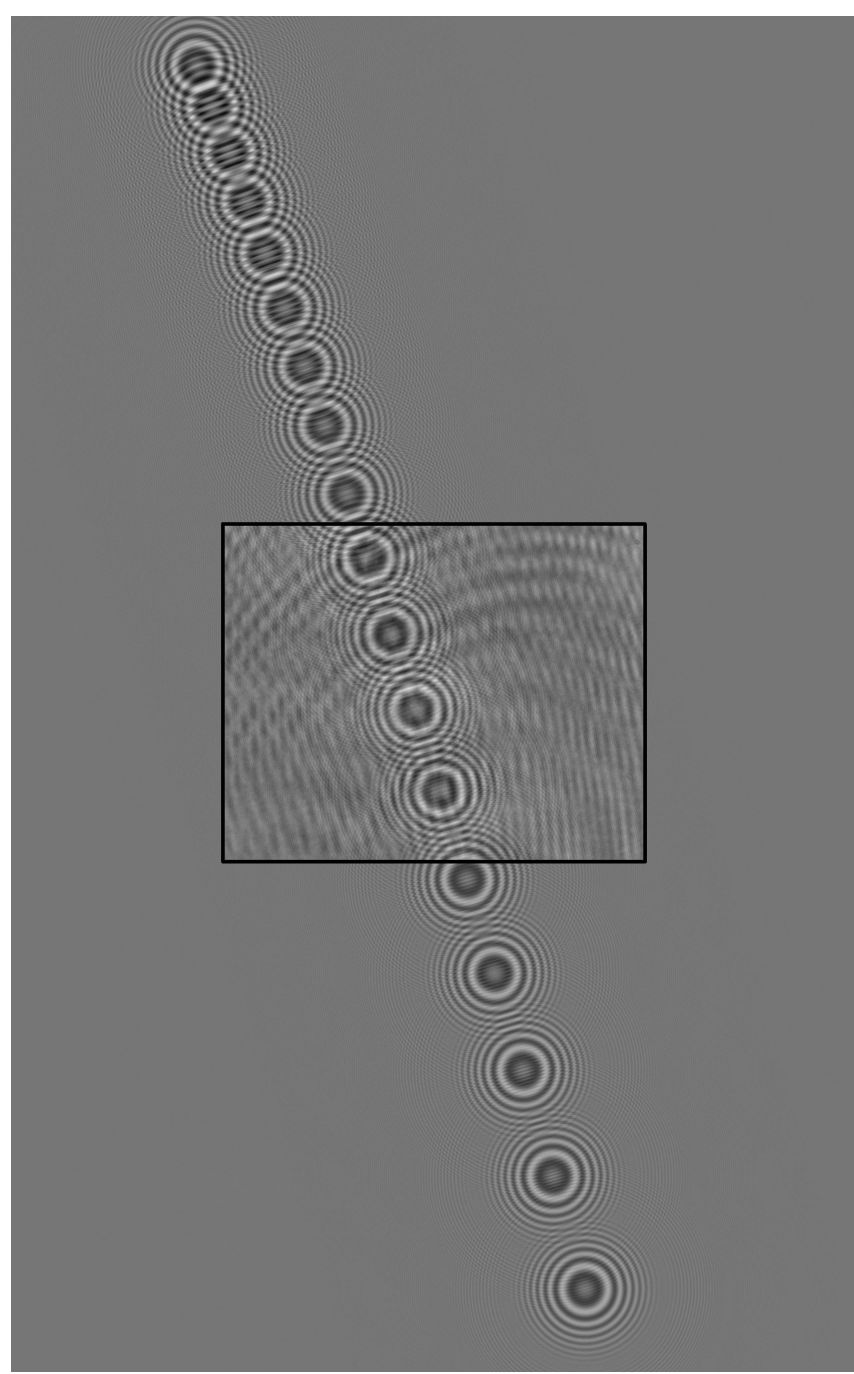

Fig. 9. Superimposition of an experimental hologram (in the box) on an synthesized hologram of 18 reconstructed particles.

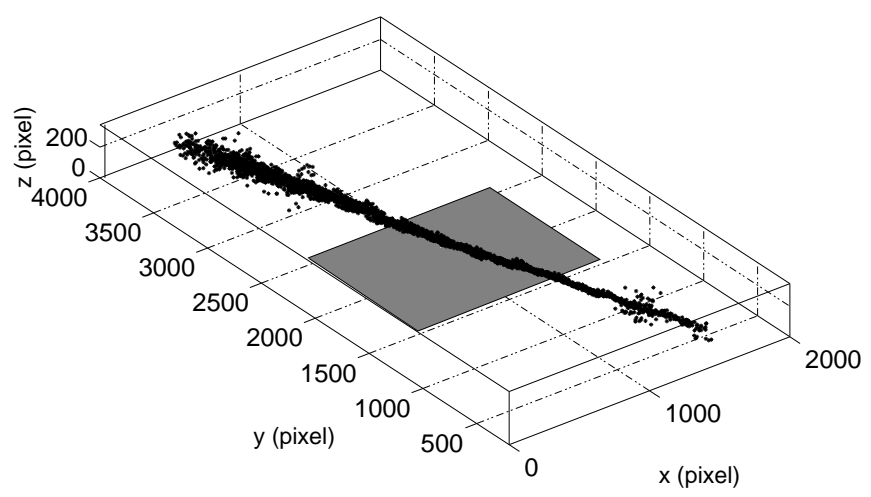

Fig. 10. Droplets jet reconstruction. 3-D representation of segmented droplets. Grey area represents the camera field of view. 


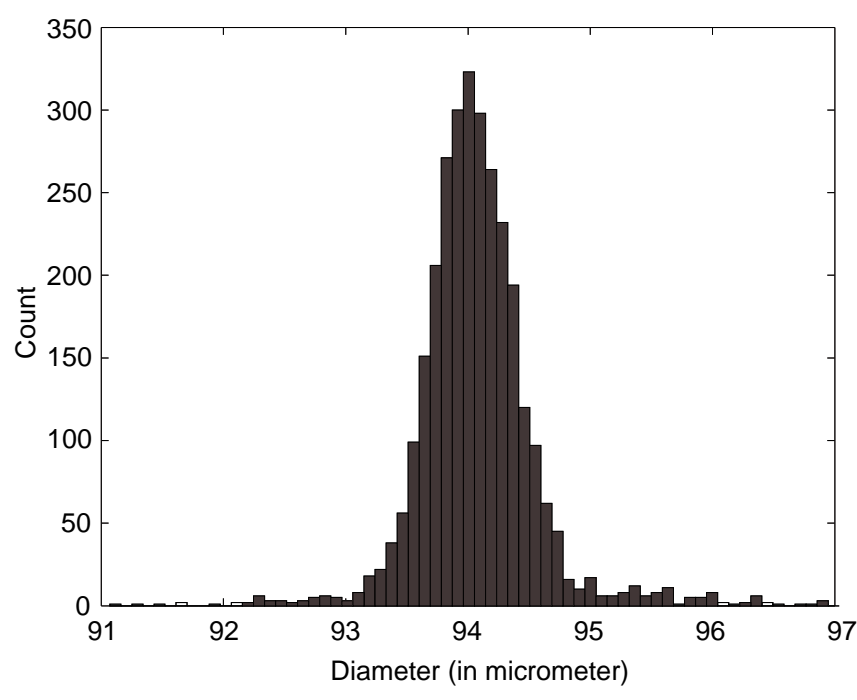

Fig. 11. Measured diameters histogram.

model and the data is minimized. The objective function turns out to have multiple local minima and thus a global optimization procedure is required to properly solve the problem. Our algorithm effectively achieves global minimization by performing an approximate detection of the particles in the whole parameter space followed by a local refinement. By repeating these steps on the residual images, our algorithm is able to detect particles even if their signature is faint compared to the speckle noise due to the other particles in the original hologram.

We have tested our algorithm on both simulated and real data. Our results show that the precision along the depth direction is greatly improved and is much better than the optical resolution in such conditions $\left(\delta z \geq \lambda / \Omega^{2}=2.6 \mathrm{~mm}\right)$. In simulations our algorithm achieves unbiased localization with constant accuracy even far from the sensor edges. Comparison with classical methods shows very strong improvements (laterally $\Delta x=\Delta y=0.3 \mu \mathrm{m}, \Delta z<1 \mu \mathrm{m}$ in depth and $\Delta r<0.05 \mu \mathrm{m}$ for radius). When dealing with experimental data, it appears that our algorithm is also robust with respect to non-homogeneous illumination and to spurious patterns due to experimental noise, as seen in Fig. 9. As the estimation is done in the data space, our algorithm can cope with truncated holograms or bad pixels: it is sufficient to set to zero the weight of unmeasured or bad pixels. Such a formulation allows us to detect particles outside the classical field of view, effectively extending the area of measurement. For instance, Fig. 9 shows that, even though only six particles signatures could be seen on the CCD, our algorithm was able to accurately localize 12 additional particles, using non-local information in the detected image.

In its current implementation, the computation burden of our algorithm is quite heavy: for our simulation, the full processing (detection, refinement and cleaning) takes about 7 minutes per particle on a Pentium IV CPU at $3.60 \mathrm{GHz}$. For routinely use, we plan to accelerate the method. This could be done if we manage to perform multiple-particle detection per pass. This improvement but also a more extensive analysis of perfomances and possible limitations of our algorithm are currently under study and will be part of forthcoming paper. For instance, as the density of particles augments, one expects that interferences between the wave diffracted by close particles to become more important which could break the approximation made in the detection step. We have checked[12] that our method works for at least 100 particles over $1000 \times 1000$ pixels but we did not tried to push this limit. Nevertheless, in-line digital holography methods are clearly devoted to diluted particle conditions (because they involve the recording of 3-D information on a 2-D detector with no phase information), the occurrence of pairs of very close particles is very unlikely in these conditions.

\section{Acknowledgments}

The authors are very grateful to the anonymous reviewers whose fruitful remarks have helped to improve the paper.

The algorithms and the simulations presented in this article have been implemented in Yorick (http://yorick.sourceforge.net), a free data processing language written by D. Munro.

\section{References}

1. T. M. Kreis, M. Adams, and W. Jüptner, "Methods of Digital Holography: A Comparison," SPIE 3098, 224-233 (1997).

2. S. Murata and N. Yasuda, "Potential of digital holography in particle measurement," Optics and Laser Technology 32, 567-574 (2000).

3. G. Pan and H. Meng, "Digital holography of particle fields: reconstruction by use of complex amplitude," Applied Optics 42, 827-833 (2003).

4. K. D. Hinsch and S. F. Herrmann, "Special issue: Holographic particle image velocimetry," Measurement Science and Technology 15 (2004).

5. F. Sheng, E. Malkiel, and J. Katz, "Digital holographic microscope for measuring three-dimensional particle distributions and motions," Applied Optics 45, 3893-3901 (2006).

6. T. Ooms, W. Koek, J. Braat, and J. Westerweel, "Optimizing Fourier filtering for digital holographic particle image velocimetry," Measurement Science and Technology 17, 304-312 (2006).

7. Y. Zhang, G. Pedrini, W. Osten, and H. Tiziani, "Applications of fractional transforms to object reconstruction from in-line holograms," Optics Letters 29(15), 1793-1795 (2004).

8. C. Buraga-Lefebvre, S. Coëtmellec, D. Lebrun, and C. Özkul, "Application of wavelet transform to hologram analysis: three-dimensional location of parti- 
cles," Optics and Lasers in Engineering 33(6), 409421 (2000).

9. M. Liebling, T. Blu, and M. Unser, "Fresnelets: New Multiresolution Wavelet Bases for Digital Holography," IEEE Transactions on image processing 12, 29-43 (2003).

10. H. Royer, "Holographic velocimetry of submicron particles," Optics Communications 20(1), 73-75 (1977).

11. C. Fournier, C. Ducottet, and T. Fournel, "Digital in-line holography: influence of the reconstruction function on the axial profile of a reconstructed particle image," Measurement Science \& Technology 15, 686-693 (2004).

12. F. Soulez, L. Denis, C. Fournier, E. Thiébaut, and
C. Goepfert, "Inverse problem approach for particle digital holography: accurate location based on local optimisation," J. Opt. Soc. Am. A 24, 1164-1171 (2007).

13. J. W. Goodman, Introduction to Fourier Optics (Mc Graw-Hill, 1996).

14. G. A. Tayler and B. J. Thompson, "Fraunhofer holography applied to particle size analysis: a reassessment," Opt. Acta. 23, 261-304 (1976).

15. L. Denis, C. Fournier, T. Fournel, C. Ducottet, and D. Jeulin, "Direct extraction of mean particle size from a digital hologram," Applied Optics 45, 944952 (2006). 